\title{
On the Iwasawa $\lambda$-invariants of real quadratic fields
}

\author{
by \\ Jae Moon Kim and Seung Ik Oh (Inchon)
}

1. Introduction. Let $k$ be a number field and $k_{\infty}=\bigcup_{n \geq 0} k_{n}$ be a $\mathbb{Z}_{p}$-extension of $k$ for a prime $p$. Let $A_{n}$ be the Sylow $p$-subgroup of the ideal class group of $k_{n}$ and $e_{n}$ be the exact power of $p$ of ${ }^{\#} A_{n}$, i.e., ${ }^{\#} A_{n}=p^{e_{n}}$. It is well known that there are integers $\mu, \lambda \geq 0$ and $\nu$, called Iwasawa invariants of $k_{\infty} / k$, such that $e_{n}=\mu p^{n}+\lambda n+\nu$ for $n \gg 0$ ([4]).

In [3], Greenberg conjectured that $\mu=\lambda=0$ if $k$ is a totally real field and gave several examples supporting the conjecture. Then in 1979, Ferrero and Washington ([1]) proved that $\mu=0$ if $k$ is an abelian field and $k_{\infty}$ is the basic $\mathbb{Z}_{p}$-extension of $k$. Since then, a lot of results have been published on the Iwasawa invariants including some recent work on the $\lambda$-invariant ([8], [12]). Greenberg's conjecture on the $\lambda$-invariant, however, still remains open even when $k$ is a real quadratic field.

In this paper we will study the $\lambda$-invariant when $k$ is a real quadratic field. One of the advantages of studying ideal class groups of abelian fields as compared with other fields is that the former have circular units which carry information about the class number. More precisely, let $E_{n}$ be the group of units and $C_{n}$ the group of circular units of $k_{n}$ defined by Sinnott ([10]). Then the index theorem of Sinnott says that ${ }^{\#} A_{n}={ }^{\#} B_{n}$ if $p$ is an odd prime, where $B_{n}$ is the Sylow $p$-subgroup of $E_{n} / C_{n}$.

From now on, we let $k$ be a real quadratic field and $k_{\infty}$ be its $\mathbb{Z}_{p^{-}}$ extension. Let $S$ be the set of primes consisting of 2 and the prime factors of the conductor and the class number of $k$. The aim of this paper is to find a criterion for the vanishing of the $\lambda$-invariant $\lambda_{p}$ of the $\mathbb{Z}_{p}$-extension $k_{\infty}$ over $k$ for $p \notin S$.

If $p$ remains inert in $k$, then $A_{n}=0$ for all $n \geq 0$ since $p \nmid h_{0}$ ([11]), and so $\lambda_{p}=0$. Thus, throughout this paper, we will always assume that $p$ splits in $k$. When $p$ splits in $k$, indivisibility of $h_{0}$ by $p$ does not imply $A_{n}=0$

2000 Mathematics Subject Classification: Primary 11R23, 11R29; Secondary 11R11.

This work was partially supported by KOSEF, 981-0101-003-2. 
for all $n \geq 0$. For instance, if $k=\mathbb{Q}(\sqrt{85})$ and $p=3$, then $A_{0}=\{0\}$ but $A_{1} \neq\{0\}([6])$. Despite this example, we can still hope that $\lambda_{3}=0$.

We briefly explain the main theorems of this paper. Let $G_{n}=\operatorname{Gal}\left(k_{n} / k\right)$ and $\Gamma=\lim _{n} G_{n}=\operatorname{Gal}\left(k_{\infty} / k\right)$. It is known that the Tate cohomology group $\widehat{H}^{0}\left(\overleftarrow{G_{n}}, C_{n}\right)$ is isomorphic to $\mathbb{Z} / p^{n} \mathbb{Z}([6])$. Let $\delta$ be a circular unit of $k$ which generates $\widehat{H}^{0}\left(G_{n}, C_{n}\right)$. We may assume that $\delta \equiv 1 \bmod p$, for otherwise we can replace $\delta$ by $\delta^{p-1}$. To be more precise, we may let $\delta=$ $\prod_{\tau \in \Delta}\left(1-\zeta_{d}^{\tau}\right)^{\chi(\tau)(p-1)}$, where $\Delta=\operatorname{Gal}\left(\mathbb{Q}\left(\zeta_{d}\right) / \mathbb{Q}\right)$ and $\zeta_{d}$ is a primitive $d$ th root of $1([6])$. Let $v_{p}$ be the $p$-adic valuation normalized by $v_{p}(p)=1$. Then we have

Theorem 1. Let $p \notin S$. If $v_{p}(\delta-1)=\max _{t}\left\{t \mid \widehat{H}^{0}\left(G_{t}, E_{t}\right)=0\right\}+1$, then $\lambda_{p}=0$.

As an application of Theorem 1, we examine the following special case. As was mentioned, ${ }^{\#} A_{n}={ }^{\#} B_{n}$. The groups $A_{n}$ and $B_{n}$ are known to be more deeply related. Indeed, as $\operatorname{Gal}\left(k_{\infty} / k\right)$-modules, $\lim A_{n}$ and $\lim B_{n}$ have the same characteristic ideals by the main conjecture which was proved by Mazur and Wiles ([7]). And it is an open question if $A_{n} \simeq B_{n}$ as abelian groups or as $G_{n}$-modules. We prove

TheOREM 2. Let $p \notin S$. Let $M$ be the integer such that $v_{p}(\delta-1)=M+1$. If ${ }^{\#} A_{M}^{G_{M}}={ }^{\#} B_{M}^{G_{M}}$, then $\lambda_{p}=0$.

Finally, in Theorem 3, we consider the $p$-adic $L$-function $L(s, \chi)$ attached to the nontrivial character $\chi$ of $k$. It is known that if $v_{p}\left(L_{p}(1, \chi)\right)=0$ (i.e., $\left.p \nmid L_{p}(1, \chi)\right)$, then $A_{n}=\{0\}$ for all $n \geq 0$ and thus $\lambda_{p}=0$ ([6]). In Theorem 3 , we generalize this.

Theorem 3. Let $p \notin S$. If $v_{p}\left(L_{p}(1, \chi)\right) \leq 1$, then $\lambda_{p}=0$.

2. Lemmas. In this section, we prove several lemmas on cohomology groups of units and circular units in the $\mathbb{Z}_{p}$-extension of a real quadratic field $k$. We keep assuming that $p$ splits in $k$ and $p \notin S$. In particular, $p$ does not divide the class number $h_{0}$ of $k$.

Lemma 1. Let $G_{n}=\operatorname{Gal}\left(k_{n} / k\right)$ and $\Gamma=\lim G_{n}=\operatorname{Gal}\left(k_{\infty} / k\right)$. Let $C_{\infty}=\bigcup_{n \geq 0} C_{n}$ and $E_{\infty}=\bigcup_{n \geq 0} E_{n}$. Then

(1) $C_{n}^{G_{n}}=C_{0}$,

$\widehat{H}^{0}\left(G_{n}, C_{n}\right) \simeq \mathbb{Z} / p^{n} \mathbb{Z}$,

$H^{1}\left(G_{n}, C_{n}\right) \simeq\left(\mathbb{Z} / p^{n} \mathbb{Z}\right)^{2}$,

$H^{1}\left(\Gamma, C_{\infty}\right) \simeq\left(\mathbb{Q}_{p} / \mathbb{Z}_{p}\right)^{2}$,

$H^{2}\left(\Gamma, C_{\infty}\right) \simeq \mathbb{Q}_{p} / \mathbb{Z}_{p}$

(2) $H^{1}\left(\Gamma, E_{\infty}\right) \simeq\left(\mathbb{Q}_{p} / \mathbb{Z}_{p}\right)^{2}$,

$H^{2}\left(\Gamma, E_{\infty}\right) \simeq \mathbb{Q}_{p} / \mathbb{Z}_{p}$ 
Pro of. For (1), we refer to [6]. In [5], Iwasawa proved that $H^{2}\left(\Gamma, E_{\infty}\right) \simeq$ $\mathbb{Q}_{p} / \mathbb{Z}_{p}$ and that $H^{1}\left(\Gamma, E_{\infty}\right)=\left(\mathbb{Q}_{p} / \mathbb{Z}_{p}\right)^{2} \oplus H$ for some finite group $H$.

Let $I_{n}$ be the ideal group of $k_{n}$ and $P_{n}$ the subgroup of $I_{n}$ generated by the principal ideals.

From $0 \rightarrow E_{n} \rightarrow k_{n}^{\times} \rightarrow P_{n} \rightarrow 0$, we have

$$
\begin{aligned}
0 \rightarrow E_{n}^{G_{n}} \rightarrow k_{n}^{\times G_{n}} \rightarrow & P_{n}^{G_{n}} \rightarrow H^{1}\left(G_{n}, E_{n}\right) \rightarrow H^{1}\left(G_{n}, k_{n}^{\times}\right) \rightarrow \\
& H^{1}\left(G_{n}, P_{n}\right) \rightarrow \widehat{H}^{0}\left(G_{n}, E_{n}\right) \rightarrow \widehat{H}^{0}\left(G_{n}, k_{n}^{\times}\right) \rightarrow \ldots
\end{aligned}
$$

Note that $E_{n}^{G_{n}} \simeq E_{0}, k_{n}^{\times G_{n}} \simeq k_{0}^{\times}$and $H^{1}\left(G_{n}, k_{n}^{\times}\right) \simeq 0$. Hence

$$
0 \rightarrow k_{0}^{\times} / E_{0} \rightarrow P_{n}^{G_{n}} \rightarrow H^{1}\left(G_{n}, E_{n}\right) \rightarrow 0
$$

and

$$
0 \rightarrow H^{1}\left(G_{n}, P_{n}\right) \rightarrow \widehat{H}^{0}\left(G_{n}, E_{n}\right) \rightarrow \widehat{H}^{0}\left(G_{n}, k_{n}^{\times}\right) \rightarrow \ldots
$$

Thus we have

$$
H^{1}\left(G_{n}, E_{n}\right) \simeq P_{n}^{G_{n}} / P_{0}
$$

and

$$
H^{1}\left(G_{n}, P_{n}\right) \simeq \operatorname{ker}\left(\widehat{H}^{0}\left(G_{n}, E_{n}\right) \rightarrow \widehat{H}^{0}\left(G_{n}, k_{n}^{\times}\right)\right) .
$$

From $0 \rightarrow P_{n} \rightarrow I_{n} \rightarrow I_{n} / P_{n} \rightarrow 0$, we have

$$
0 \rightarrow P_{n}^{G_{n}} \rightarrow I_{n}^{G_{n}} \rightarrow\left(I_{n} / P_{n}\right)^{G_{n}} \rightarrow H^{1}\left(G_{n}, P_{n}\right) \rightarrow H^{1}\left(G_{n}, I_{n}\right) \simeq 0 \rightarrow \ldots
$$

Hence

$$
0 \rightarrow P_{n}^{G_{n}} / P_{0} \rightarrow I_{n}^{G_{n}} / P_{0} \rightarrow\left(I_{n} / P_{n}\right)^{G_{n}} \rightarrow H^{1}\left(G_{n}, P_{n}\right) \rightarrow 0 .
$$

Therefore

$$
\begin{aligned}
0 \rightarrow H^{1}\left(G_{n}, E_{n}\right) \rightarrow I_{n}^{G_{n}} / P_{0} \rightarrow & \left(I_{n} / P_{n}\right)^{G_{n}} \rightarrow \\
& \operatorname{ker}\left(\widehat{H}^{0}\left(G_{n}, E_{n}\right) \rightarrow \widehat{H}^{0}\left(G_{n}, k_{n}^{\times}\right)\right) \rightarrow 0 .
\end{aligned}
$$

We also have an exact sequence

$$
0 \rightarrow I_{0} / P_{0} \rightarrow I_{n}^{G_{n}} / P_{0} \rightarrow I_{n}^{G_{n}} / I_{0} \rightarrow 0 .
$$

By tensoring the above sequence with $\mathbb{Z}_{p}$, we get

$$
I_{n}^{G_{n}} / P_{0} \otimes \mathbb{Z}_{p} \simeq I_{n}^{G_{n}} / I_{0} \otimes \mathbb{Z}_{p} \simeq I_{n}^{G_{n}} / I_{0} \simeq\left(\mathbb{Z} / p^{n} \mathbb{Z}\right)^{2}
$$

since $p \nmid h_{0}$. Therefore, we obtain

$$
\begin{aligned}
0 \rightarrow H^{1}\left(G_{n}, E_{n}\right) \rightarrow I_{n}^{G_{n}} / I_{0} & \rightarrow\left(I_{n} / P_{n}\right)^{G_{n}} \rightarrow \\
& \operatorname{ker}\left(\widehat{H}^{0}\left(G_{n}, E_{n}\right) \rightarrow \widehat{H}^{0}\left(G_{n}, k_{n}^{\times}\right)\right) \rightarrow 0 .
\end{aligned}
$$


For $m>n$, we have a commutative diagram

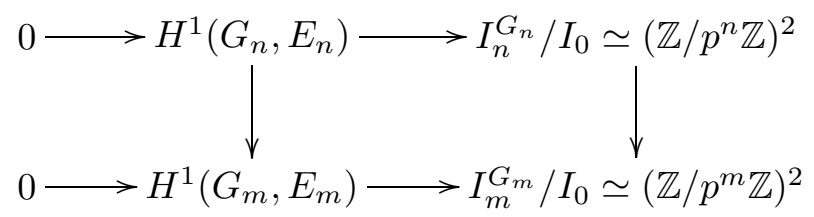

By taking direct limits, we have

$$
0 \rightarrow \underline{\lim _{\longrightarrow}} H^{1}\left(G_{n}, E_{n}\right) \simeq\left(\mathbb{Q}_{p} / \mathbb{Z}_{p}\right)^{2} \oplus H \rightarrow \underline{\lim }\left(\mathbb{Z} / p^{n} \mathbb{Z}\right)^{2} \simeq\left(\mathbb{Q}_{p} / \mathbb{Z}_{p}\right)^{2} .
$$

Hence $H$ must be trivial, and so

$$
H^{1}\left(\Gamma, E_{\infty}\right) \simeq\left(\mathbb{Q}_{p} / \mathbb{Z}_{p}\right)^{2} .
$$

Lemma 2. The induced homomorphism $H^{1}\left(\Gamma, C_{\infty}\right) \rightarrow H^{1}\left(\Gamma, E_{\infty}\right)$ is surjective.

Proof. Let $B_{n}=E_{n} / C_{n} \otimes \mathbb{Z}_{p}$ be the Sylow $p$-subgroup of $E_{n} / C_{n}$. It is known ([2]) that the natural map $E_{n} / C_{n} \rightarrow E_{m} / C_{m}$ for $m>n$ is injective. Let $B_{\infty}=\lim _{n} B_{n}$ be the direct limit under the natural injection. So $B_{\infty}=$ $\lim _{\longrightarrow}\left(E_{n} / C_{n} \otimes \mathbb{Z}_{p}\right)=E_{\infty} / C_{\infty} \otimes \mathbb{Z}_{p}$. From $0 \rightarrow C_{\infty} \rightarrow E_{\infty} \rightarrow E_{\infty} / C_{\infty} \rightarrow 0$, we have $0 \rightarrow C_{\infty} \otimes \mathbb{Z}_{p} \rightarrow E_{\infty} \otimes \mathbb{Z}_{p} \rightarrow B_{\infty} \rightarrow 0$. Then we obtain a long exact sequence

$$
\begin{array}{r}
0 \rightarrow B_{\infty}^{\Gamma} / B_{0} \rightarrow H^{1}\left(\Gamma, C_{\infty}\right) \rightarrow H^{1}\left(\Gamma, E_{\infty}\right) \rightarrow H^{1}\left(\Gamma, B_{\infty}\right) \rightarrow H^{2}\left(\Gamma, C_{\infty}\right) \\
\rightarrow H^{2}\left(\Gamma, E_{\infty}\right) \rightarrow H^{2}\left(\Gamma, B_{\infty}\right) \rightarrow \ldots
\end{array}
$$

Note that $B_{\infty}^{\Gamma_{n}}$ is finite ([11], Lemma 15.39, Proposition 15.44), where $\Gamma_{n}=$ $\operatorname{Gal}\left(k_{\infty} / k_{n}\right)$. Thus $B_{\infty}^{\Gamma}$ is also finite. Also note that $H^{2}\left(\Gamma, B_{\infty}\right)=\{0\}$, since $B_{\infty}$ is a torsion group ([9], Proposition 3.25, Example 17). Hence the above long exact sequence reads

$$
0 \rightarrow \text { finite } \rightarrow\left(\mathbb{Q}_{p} / \mathbb{Z}_{p}\right)^{2} \rightarrow\left(\mathbb{Q}_{p} / \mathbb{Z}_{p}\right)^{2} \rightarrow H^{1}\left(\Gamma, B_{\infty}\right) \rightarrow \mathbb{Q}_{p} / \mathbb{Z}_{p} \rightarrow \mathbb{Q}_{p} / \mathbb{Z}_{p} \rightarrow 0
$$

Therefore $H^{1}\left(\Gamma, B_{\infty}\right)$ is a finite group. Since $\left(\mathbb{Q}_{p} / \mathbb{Z}_{p}\right)^{2}$ has no nontrivial cokernel, $H^{1}\left(\Gamma, C_{\infty}\right) \rightarrow H^{1}\left(\Gamma, E_{\infty}\right)$ is surjective.

Lemma 3. The induced maps $H^{1}\left(G_{n}, C_{n}\right) \rightarrow H^{1}\left(G_{n}, E_{n}\right)$ and $\widehat{H}^{0}\left(G_{n}, C_{n}\right)$ $\rightarrow \widehat{H}^{0}\left(G_{n}, E_{n}\right)$ are surjective for all $n \geq 1$.

Proof. From $0 \rightarrow C_{n} \rightarrow E_{n} \rightarrow E_{n} / C_{n} \rightarrow 0$, we get a long exact sequence

$$
0 \rightarrow B_{0} \rightarrow B_{n}^{G_{n}} \rightarrow H^{1}\left(G_{n}, C_{n}\right) \rightarrow H^{1}\left(G_{n}, E_{n}\right) \stackrel{f}{\rightarrow} H^{1}\left(G_{n}, B_{n}\right) \rightarrow .
$$

Since $p \nmid h_{0}$, we have $B_{0}=\{0\}$. Then consider the following commutative 
diagram:

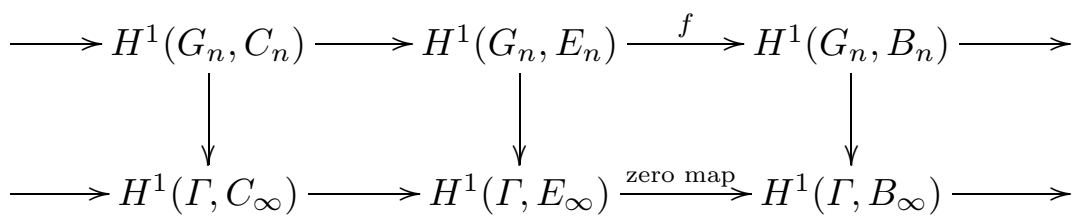

where vertical maps are inflations. From the injectivity of the inflation map $H^{1}\left(G_{n}, B_{n}\right) \rightarrow H^{1}\left(\Gamma, B_{\infty}\right)$, one can easily see that $f$ is a zero map. Hence $H^{1}\left(G_{n}, C_{n}\right) \rightarrow H^{1}\left(G_{n}, E_{n}\right)$ is surjective. The surjectivity of $\widehat{H}^{0}\left(G_{n}, C_{n}\right) \simeq$ $C_{0} / N_{n} C_{n} \rightarrow \widehat{H}^{0}\left(G_{n}, E_{n}\right) \simeq E_{0} / N_{n} E_{n}$ follows immediately from the assumption $p \nmid h_{0}$, where $N_{n}$ is the norm map from $k_{n}$ to $k_{0}$.

3. $\lambda$-invariant. In this section, we will prove the main theorems stated in the introduction. Since $p$ splits in $k$, the completion of $k$ at a prime $\wp$ above $p$ is $\mathbb{Q}_{p}$. We denote the completion of $k_{n}$ at the prime of $k_{n}$ above $\wp$ by $\mathbb{Q}_{p, n}$. Let $\delta=\prod_{\tau \in \Delta}\left(1-\zeta_{d}^{\tau}\right)^{\chi(\tau)(p-1)}$ be a circular unit of $k$ which generates $\widehat{H}^{0}\left(G_{n}, C_{n}\right)$ as in the introduction.

Theorem 1. Let $p \notin S$. If $v_{p}(\delta-1)=\max _{t}\left\{t \mid \widehat{H}^{0}\left(G_{t}, E_{t}\right)=0\right\}+1$, then $\lambda_{p}=0$.

REMARK. Since $N_{m} E_{m} \subset N_{n} E_{n}$ for $m>n, \widehat{H}^{0}\left(G_{n}, E_{n}\right)$ is a quotient group of $\widehat{H}^{0}\left(G_{m}, E_{m}\right)$. Thus if $\widehat{H}^{0}\left(G_{m}, E_{m}\right)=0$, then $\widehat{H}^{0}\left(G_{n}, E_{n}\right)=0$. And since $E_{0} / \bigcap_{n \geq 0} N_{n} E_{n} \simeq \mathbb{Q}_{p} / \mathbb{Z}_{p}$ (we are assuming that $p$ splits in $k$ ), $\widehat{H}^{0}\left(G_{m}, E_{m}\right) \neq 0$ for sufficiently large $m$. Thus $\max _{t}\left\{t \mid \widehat{H}^{0}\left(G_{t}, E_{t}\right)=0\right\}$ is well defined. Also note that $\widehat{H}^{0}\left(G_{t}, E_{t}\right)$ is generated by $\delta$ since $\widehat{H}^{0}\left(G_{t}, C_{t}\right) \rightarrow$ $\widehat{H}^{0}\left(G_{t}, E_{t}\right)$ is surjective. Hence, if $\widehat{H}^{0}\left(G_{t}, E_{t}\right)=\{0\}$, then $\delta=N_{t}\left(\eta_{t}\right)$ for some $\eta_{t} \in k_{t}$. By reading this equation in the completion, i.e., $\eta_{t} \in \mathbb{Q}_{p, t}$ and $N_{t}=N_{\mathbb{Q}_{p, t} / \mathbb{Q}_{p}}$, we get $\delta \equiv 1 \bmod p^{t+1}$. Therefore $v_{p}(\delta-1) \geq$ $\max _{t}\left\{t \mid \widehat{H}^{0}\left(G_{t}, E_{t}\right)=0\right\}+1$.

Proof of Theorem 1. We will prove the vanishing of $\lambda_{p}$ by showing that every ideal in $k_{n}$ capitulates in $k_{\infty}$. Let $D_{n}$ be the kernel of the natural

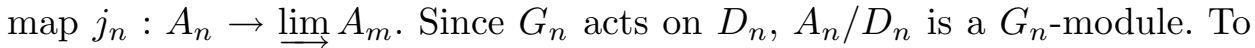
prove $A_{n} / D_{n}=\{0\}$, it is enough to show that $\left(A_{n} / D_{n}\right)^{G_{n}}=\{0\}$. Let $C$ be a class in $A_{n}$ such that $C^{\sigma-1} \in D_{n}$, where $\sigma$ is a topological generator of $\Gamma=\operatorname{Gal}\left(k_{\infty} / k\right)$. Thus $C^{\sigma-1}=0$ in $A_{m}$ for $m \gg n$. Let $j_{n, m}: A_{n} \rightarrow A_{m}$ be the natural map and write $j_{n, m}(C)=C^{\prime}$. Let $\mathfrak{a}$ be an ideal of $k_{m}$ which represents $C^{\prime}$. Thus $\mathfrak{a}^{\sigma-1}=(\alpha)$ for some $\alpha \in k_{m}$.

Since $\left(N_{m}(\alpha)\right)=N_{m} \mathfrak{a}^{\sigma-1}=(1), N_{m}(\alpha)=\varepsilon$ is a unit in $k$. Let $a, 0 \leq a<$ $p^{m}$, be such that $\varepsilon \bmod N_{m} E_{m}=\delta^{a} \bmod N_{m} E_{m}$. That is, $\varepsilon=\delta^{a} N_{m}\left(\eta_{m}\right)$ 
for some unit $\eta_{m} \in E_{m}$. This is possible by Lemma 3. Then $\delta^{a}=N_{m}\left(\alpha \eta_{m}^{-1}\right)$. Thus $\delta^{a}$ is a norm from $\mathbb{Q}_{p, m}$ to $\mathbb{Q}_{p}$. Therefore $\delta^{a} \equiv 1 \bmod p^{m+1}$.

Let $M=\max _{t}\left\{t \mid \widehat{H}^{0}\left(G_{t}, E_{t}\right)=0\right\}=v_{p}(\delta-1)-1$. So $\delta \equiv 1 \bmod$ $p^{M+1}$ but $\delta \not \equiv 1 \bmod p^{M+2}$. Since $\delta^{a} \equiv 1 \bmod p^{m+1}$, we must have $a \equiv 0$ $\bmod p^{m-M}$. Let $a=p^{m-M} b$. Then $\varepsilon=\delta^{a} N_{m}\left(\eta_{m}\right)=\left(\delta^{b}\right)^{p^{m-M}} N_{m}\left(\eta_{m}\right)$. Since $\widehat{H}^{0}\left(G_{M}, E_{M}\right)=0, \delta^{b}=N_{M}\left(\eta_{M}\right)$ for some $\eta_{M} \in E_{M}$. Hence $\delta^{a}=$ $\left(\delta^{b}\right)^{p^{m-M}}=N_{m}\left(\eta_{M}\right)$, and thus $\varepsilon=N_{m}\left(\eta_{M} \eta_{m}\right)$. Therefore $\varepsilon=N_{m}(\alpha)=$ $N_{m}(\eta)$ for a unit $\eta=\eta_{M} \eta_{m} \in E_{m}$. So $\alpha=\eta \beta^{1-\sigma}$ for some $\beta \in k_{m}$. Hence $\mathfrak{a}^{\sigma-1}=(\alpha)=(\beta)^{1-\sigma}$, which means that $\mathfrak{a}(\beta)$ is fixed under $G_{m}$. Thus $\mathfrak{a}(\beta)$ is a product of ideals from $k_{0}$ and primes above $p$. Since primes above $p$ capitulate, $j_{m}\left(C^{\prime}\right)=0$. Therefore $j_{n}(C)=0$. This completes the proof.

Theorem 2. Let $p \notin S$. Let $M$ be the integer such that $v_{p}(\delta-1)=M+1$. If ${ }^{\#} A_{M}^{G_{M}}={ }^{\#} B_{M}^{G_{M}}$, then $\lambda_{p}=0$.

Proof. By the remark after Theorem 1, it is enough to show that $v_{p}(\delta-1) \leq \max _{t}\left\{t \mid \widehat{H}^{0}\left(G_{t}, E_{t}\right)=0\right\}+1$. Thus it suffices to show that $\widehat{H}^{0}\left(G_{M}, E_{M}\right)=\{0\}$.

Since $\delta \equiv 1 \bmod p^{M+1}, \delta=N_{\mathbb{Q}_{p, M} / \mathbb{Q}_{p}}(\eta)$ for some unit $\eta$ in $\mathbb{Q}_{p, M}$. Since primes of $k$ above $q(q \neq p)$ are unramified in $k_{M}, \delta$ is a local norm for all primes. Therefore $\delta$ is a global norm by the Hasse theorem. That is, $\widehat{H}^{0}\left(G_{M}, C_{M}\right) \rightarrow \widehat{H}^{0}\left(G_{M}, k_{M}^{*}\right)$ is a zero map. Since $\widehat{H}^{0}\left(G_{M}, C_{M}\right) \rightarrow$ $\widehat{H}^{0}\left(G_{M}, E_{M}\right)$ is surjective by Lemma $3, \widehat{H}^{0}\left(G_{M}, E_{M}\right) \rightarrow \widehat{H}^{0}\left(G_{M}, k_{M}^{*}\right)$ is a zero map. Hence $\operatorname{ker}\left(\widehat{H}^{0}\left(G_{M}, E_{M}\right) \rightarrow \widehat{H}^{0}\left(G_{M}, k_{M}^{*}\right)\right)=\widehat{H}^{0}\left(G_{M}, E_{M}\right)$. Thus from a sequence similar to $(*)$ in Section 2 , we have an exact sequence

$$
0 \rightarrow H^{1}\left(G_{M}, E_{M}\right) \rightarrow I_{M}^{G_{M}} / I_{0} \rightarrow A_{M}^{G_{M}} \rightarrow \widehat{H}^{0}\left(G_{M}, E_{M}\right) \rightarrow 0 .
$$

Since the Herbrand quotient for $E_{M}$ is $p^{M}$, we have

$$
{ }^{\#} H^{1}\left(G_{M}, E_{M}\right) /{ }^{\#} \widehat{H}^{0}\left(G_{M}, E_{M}\right)=p^{M} .
$$

Since ${ }^{\#} I_{M}^{G_{M}} / I_{0}=p^{2 M}$, we get ${ }^{\#} A_{M}^{G_{M}}=p^{M}$.

By Lemma 3, we have another exact sequence

$$
0 \rightarrow B_{M}^{G_{M}} \rightarrow H^{1}\left(G_{M}, C_{M}\right) \rightarrow H^{1}\left(G_{M}, E_{M}\right) \rightarrow 0 .
$$

Note that ${ }^{\#} B_{M}^{G_{M}}={ }^{\#} A_{M}^{G_{M}}=p^{M}$ and that ${ }^{\#} H^{1}\left(G_{M}, C_{M}\right)=p^{2 M}$. Therefore ${ }^{\#} H^{1}\left(G_{M}, E_{M}\right)=p^{M}$, and so ${ }^{\#} \widehat{H}^{0}\left(G_{M}, E_{M}\right)=0$.

To prove Theorem 3 , we need a lemma. We let $\delta=\prod_{\tau \in \Delta}\left(1-\zeta_{d}^{\tau}\right)^{\chi(\tau)(p-1)}$ as before.

Lemma 4. If $p \mid L_{p}(1, \chi)$, then $\widehat{H}^{0}\left(G_{1}, E_{1}\right)=\{0\}$.

Proof. Consider the exact sequence $0 \rightarrow B_{1}^{G_{1}} \rightarrow H^{1}\left(G_{1}, C_{1}\right) \rightarrow$ $H^{1}\left(G_{1}, E_{1}\right) \rightarrow 0$. Since $p \mid L_{p}(1, \chi), B_{1} \neq\{0\}\left([6]\right.$ or [8]) and so $B_{1}^{G_{1}} \neq\{0\}$. 
Since $H^{1}\left(G_{1}, C_{1}\right) \simeq \mathbb{Z} / p \mathbb{Z} \times \mathbb{Z} / p \mathbb{Z}$, we see that $H^{1}\left(G_{1}, E_{1}\right)$ is either $\{0\}$ or $\mathbb{Z} / p \mathbb{Z}$. But $\pi^{\sigma-1}$ is a nontrivial element of $H^{1}\left(G_{1}, E_{1}\right)$, where $\pi$ is a prime element of $\mathbb{Q}_{1}$, the subextension of $\mathbb{Q}\left(\zeta_{p^{2}}\right)$ of degree $p$ over $\mathbb{Q}$. Thus $H^{1}\left(G_{1}, E_{1}\right) \simeq \mathbb{Z} / p \mathbb{Z}$. Since the Herbrand quotient for $E_{1}$ is $p$, we have $\widehat{H}^{0}\left(G_{1}, E_{1}\right)=\{0\}$.

Theorem 3. Let $p \notin S$. If $v_{p}\left(L_{p}(1, \chi)\right) \leq 1$, then $\lambda_{p}=0$.

Proof. If $v_{p}\left(L_{p}(1, \chi)\right)=0$, then there is nothing to prove as was explained in the introduction. So we assume that $v_{p}\left(L_{p}(1, \chi)\right)=1$. Let $\delta$ be as above. Note that

$$
\begin{aligned}
L_{p}(1, \chi) & =-\left(1-\frac{1}{p}\right) \frac{g(\chi)}{d} \sum_{\tau \in \Delta} \chi(\tau) \log _{p}\left(1-\zeta_{d}^{\tau}\right) \\
& =-\frac{g(\chi)}{p d} \log _{p}\left(\prod_{\tau \in \Delta}\left(1-\zeta_{d}^{\tau}\right)^{\chi(\tau)(p-1)}\right)=-\frac{g(\chi)}{p d} \log _{p} \delta,
\end{aligned}
$$

where $g(\chi)=\sum_{a=1}^{d} \chi(a) \zeta_{d}^{a}$ is the Gauss sum for $\chi$. Thus $v_{p}\left(\log _{p} \delta\right)=$ $v_{p}\left(L_{p}(1, \chi)\right)+1=2$.

Since $\delta \equiv 1 \bmod p$, we have $v_{p}\left(\log _{p} \delta\right)=v_{p}(\delta-1)$. Therefore $v_{p}(\delta-1)=2$. By Lemma $4, \widehat{H}^{0}\left(G_{1}, E_{1}\right)=\{0\}$. But $\widehat{H}^{0}\left(G_{2}, E_{2}\right)$ is not trivial, for otherwise $v_{p}(\delta-1) \geq 2+1=3$ by the Remark after Theorem 1 . Hence $v_{p}(\delta-1)=$ $2=\max _{t}\left\{t \mid \widehat{H}^{0}\left(G_{t}, E_{t}\right)=0\right\}+1$, and thus $\lambda_{p}=0$ by Theorem 1 .

\section{References}

[1] B. Ferrero and L. Washington, The Iwasawa invariant $\mu_{p}$ vanishes for abelian number fields, Ann. of Math. 109 (1979), 377-395.

[2] R. Gold and J. M. Kim, Bases for cyclotomic units, Compositio Math. 71 (1989), $13-28$.

[3] R. Greenberg, On the Iwasawa invariants of totally real number fields, Amer. J. Math. 98 (1976), 263-284.

[4] K. Iwasawa, On $\mathbb{Z}_{l}$-extensions of algebraic number fields, Ann. of Math. 98 (1973), 246-326.

[5] -, On cohomology groups of units for $\mathbb{Z}_{p}$-extensions, Amer. J. Math. 105 (1983), 189-200.

[6] J. M. Kim, Class numbers of real quadratic fields, Bull. Austral. Math. Soc. 57 (1998), 261-274.

[7] B. Mazur and A. Wiles, Class fields of abelian extensions of $\mathbb{Q}$, Invent. Math. 76 (1984), 179-330.

[8] M. Ozaki, The class group of $\mathbb{Z}_{p}$-extensions over totally real number fields, Tôhoku Math. J. 49 (1997), 431-435.

[9] A. N. Parshin and I. R. Shafarevich, Number Theory II, Encyclopaedia Math. Sci. 62, Springer, 1992.

[10] W. Sinnott, On the Stickelberger ideal and the circular units of an abelian field, Invent. Math. 62 (1980), 181-234. 
[11] L. Washington, Introduction to Cyclotomic Fields, 2nd ed., Grad. Texts in Math. 83, Springer, New York, 1996.

[12] G. Yamamoto, On the vanishing of Iwasawa invariants of certain $(p, p)$-extensions of $\mathbb{Q}$, Proc. Japan Acad. Ser. A 73 (1997), no. 3, 45-47.

Department of Mathematics

Inha University, Inchon, Korea

E-mail: jmkim@math.inha.ac.kr

sioh@math.inha.ac.kr

Received on 1.3.1999

and in revised form 16.3.2000 